\title{
Nonlinear Stability of Periodic Traveling Wave Solutions for $(n+1)$-Dimensional Coupled Nonlinear Klein-Gordon Equations
}

\author{
Cong Sun ${ }^{1}$ and Bo Jiang ${ }^{2}$ \\ ${ }^{1}$ College of Mathematics, Jilin University, Changchun 130012, China \\ ${ }^{2}$ The City College of Jilin Jianzhu University, Changchun 130111, China \\ Correspondence should be addressed to Cong Sun; 3211519@163.com
}

Received 20 March 2015; Accepted 26 July 2015

Academic Editor: Gunther Brenner

Copyright (c) 2015 C. Sun and B. Jiang. This is an open access article distributed under the Creative Commons Attribution License, which permits unrestricted use, distribution, and reproduction in any medium, provided the original work is properly cited.

We study the existence and orbital stability of smooth periodic traveling waves solutions of the $(n+1)$-dimensional coupled nonlinear Klein-Gordon equations. Such a system occurs in quantum mechanics, fluid mechanics, and optical fiber communication. Inspired by Angulo Pava's results (2007), and by applying the stability theory established by Grillakis et al. (1987), we prove the existence of periodic traveling waves solutions and obtain the orbital stability of the solutions to this system.

\section{Introduction}

This paper pays close attention to $(n+1)$-dimensional system of coupled nonlinear Klein-Gordon equations

$$
\begin{aligned}
\phi_{t t}-\Delta \phi+m_{1} \phi & =\left(a_{11}|\phi|^{2}+a_{12}|\psi|^{2}\right) \phi, \\
\psi_{t t}-\Delta \psi+m_{2} \psi & =\left(a_{21}|\phi|^{2}+a_{22}|\psi|^{2}\right) \psi,
\end{aligned}
$$

where $\Delta=\partial^{2} / \partial x_{1}^{2}+\partial^{2} / \partial x_{2}^{2}+\cdots+\partial^{2} / \partial x_{n}^{2}$ is Laplace operator, $x \in R^{n}, m_{1}<0, m_{2}<0, a_{i j} \in R(i, j=1,2), a_{11} \neq a_{21}$, and $\phi(x, t)$ and $\psi(x, t)$ are complex functions.

Equation (1) frequently describes physical questions, for example, crystal growths and dislocations. In recent decades, lots of methods have been put forward, such as the direct integral method, the hyperbolic function expansion method $[1,2]$, mixing exponential method, and Jacobi elliptic function expansion method $[3,4]$, to obtain the exact solutions of nonlinear evolution equations.

In this paper, we are interested in the existence of a smooth periodic solution and the orbital stability of solution of (1). A comprehensive development of stability results for this type has been acquired by Grillakis et al. [5, 6], Benjamin [7], and Weinstein [8]. Moreover, many results of orbital stability of Klein-Gordon equation have been attained. For example, Shatah [9] has given sufficient conditions of orbital stability in such a case:

$$
u_{t t}-\Delta u+u+g(u) \arg u=0, \quad t \in R, x \in R^{n},
$$

and it is showed that the standing wave solutions $e^{\text {ict }} \phi(x)$ of this equation have orbital stability when $g(u) \arg u=$ $-|u|^{p-1} u$, with $p>1$ as an integer. In addition, Ohta and Todorova [10] have established the instability of (2) when $1+4 / n<p<1+4 /(n-2)$. In this case, we say that this wave $e^{i c t} \phi(x)$ is unstable for (2); suppose for any $\varepsilon>0$, there exists $\left(u_{0}, u_{1}\right) \in X=H^{1}(R) \times L^{2}(R)$ such that $\left\|\left(u_{0}, u_{1}\right)-(\phi, i c \phi)\right\|_{X}<$ $\varepsilon$ and the solution $u(t)=\left(u, u_{t}\right)$ of $(2)$ with $u(0)=\left(u_{0}, u_{1}\right)$ satisfies $\lim _{t \rightarrow \infty}\|u(t)\|_{X}=\infty$. Moreover, Angulo Pava [11] has studied the orbital stability of cnoidal waves solution of the following system:

$$
\begin{aligned}
u_{t}-a u_{x x x}+6 u_{x} u & =2 b v v_{x}, \\
v_{t}+v_{x x x}+3 u v_{x} & =0,
\end{aligned}
$$

where $b>0$, and he obtained the orbital stability of the cnoidal solutions in the energy space $H_{\text {per }}^{1}(0, L) \times H_{\text {per }}^{1}([0, L])$ with regard to the periodic flow of (3) when $a \geq 1 / 20$.

In our paper, we work on the existence and orbital stability of periodic traveling waves solution of system (1). The plan of the paper is as follows: in Section 2, we obtain the existence of 
periodic traveling waves solution of system (1). In Section 3, the spectrum of operator $L$ (see (26)) is studied in detail. In Section 4, the orbital stability of system (1) is established. In Section 5, we give a brief discussion and conclusion to state the application of system (1) in the field of engineering.

Notation. Let $L^{p}(\Omega)$ denote Lebesgue measurable space for the open subset $\Omega$ in $R$, the norm $\|f\|_{L^{p}}^{p}=\int_{\Omega}|f|^{p} d x$, and $p \geq$ 1. $X=H^{1}(R) \times H^{1}(R) \times H^{1}(R) \times H^{1}(R)$ denotes the complex space with real inner product $\left(u_{1}, u_{2}\right)=\operatorname{Re} \int\left(u_{1} \overline{u_{2}}+u_{1 x} \overline{u_{2 x}}\right)$. The dual space of $X$ is $X^{*}=H^{-1}(R) \times H^{-1}(R) \times H^{-1}(R) \times$ $H^{-1}(R)$. There exists a natural isomorphism $I: X \rightarrow X^{*}$ denoted by $\langle$ Iu, $v\rangle=(u, v)$. Here,

$$
\begin{aligned}
\langle u, v\rangle & =\int_{R} u \bar{v} d x, \\
I & =\left(\begin{array}{cccc}
-\frac{\partial^{2}}{x^{2}}+1 & & \\
& 1 & & \\
& & 1 & \\
& & & 1
\end{array}\right) .
\end{aligned}
$$

\section{Existence of the Periodic Traveling Wave Solution for the KG Equations}

In this section, motivated by Angulo Pava's work [12], we will study the existence of a smooth curve of periodic traveling waves solutions to the coupled nonlinear KleinGordon equations (1) with the form

$$
\begin{aligned}
\phi(x, t) & =e^{i \eta_{1}} u(\xi), \\
\psi(x, t) & =e^{i \eta_{2}} \varphi(\xi), \\
\eta_{1} & =\sum_{j=1}^{n} \alpha_{j} x_{j}-c t, \\
\eta_{2} & =\sum_{j=1}^{n} \beta_{j} x_{j}-c t,
\end{aligned}
$$

where $\xi=\sum_{j=1}^{n} k_{j} x_{j}, k_{j}, \alpha_{j}, \beta_{j} \in R, j=1,2, \ldots, n$, and $x=$ $\left(x_{1}, x_{2}, \ldots, x_{n}\right)$. The profile $(u(\xi), \varphi(\xi))$ satisfies the boundary conditions $|u(\xi)| \rightarrow 0$ and $|\varphi(\xi)| \rightarrow 0$ as $|\xi| \rightarrow \infty$ and the constant $c$ denotes the speed of traveling waves.

By substituting (5) in (1), we obtain

$$
\begin{aligned}
& -c^{2} u-\sum_{i=1}^{n} k_{i}^{2} u^{\prime \prime}+u \sum_{j=1}^{n} \alpha_{j}^{2}+m_{1} u=\left(a_{11} u^{2}+a_{12} \varphi^{2}\right) u, \\
& -c^{2} \varphi-\sum_{i=1}^{n} k_{i}^{2} \varphi^{\prime \prime}+\varphi \sum_{j=1}^{n} \beta_{j}^{2}+m_{2} \varphi=\left(a_{21} u^{2}+a_{22} \varphi^{2}\right) \varphi
\end{aligned}
$$

Now, we consider the the special solutions $(u(\xi), \varphi(\xi))=$ $(a \varphi(\xi), \varphi(\xi))$ of (6) where $a \in R^{-}$. Then, (6) is transformed into

$$
\begin{gathered}
\varphi^{\prime \prime}-B_{0} \varphi-\varphi^{3} \frac{\left(-a_{11} a^{2}-a_{12}\right)}{\sum_{i=1}^{n} k_{i}^{2}}=0, \\
\varphi^{\prime \prime}-B_{0}^{\prime} \varphi-\varphi^{3} \frac{\left(-a_{21} a^{2}-a_{22}\right)}{\sum_{i=1}^{n} k_{i}^{2}}=0,
\end{gathered}
$$

where $B_{0}=\left(\alpha+m_{1}-c^{2}\right) / \sum_{i=1}^{n} k_{i}^{2}$ and $B_{0}^{\prime}=\left(\beta+m_{2}-\right.$ $\left.c^{2}\right) / \sum_{i=1}^{n} k_{i}^{2}$. Moreover, we assume that $-m_{1}+c^{2}-\alpha>0$ and $-m_{2}+c^{2}-\beta>0$.

Notice the relation $u=a \varphi$; hence, the corresponding parameters satisfy the relations $a^{2}=\left(a_{22}-a_{12}\right) /\left(a_{11}-a_{21}\right)$ and $B_{0}=B_{0}^{\prime}$.

Next, to study the solutions of (7), we only need to consider the first equation of (7):

$$
\varphi^{\prime \prime}-B_{0} \varphi-m \varphi^{3}=0 .
$$

Firstly, we suppose that $B_{0}>0$ and $m=\left(-a_{11} a^{2}-a_{12}\right) /$ $\sum_{i=1}^{n} k_{i}^{2}<0$.

Multiplying (8) by $\varphi^{\prime}$ and integrating once, we obtain

$$
\left(\varphi^{\prime}\right)^{2}=-\frac{m}{2}\left[\left(P_{1}^{2}-\varphi^{2}\right)\left(\varphi^{2}-Q_{1}^{2}\right)\right],
$$

where $h$ is a constant of integration, $P_{1}^{2}=-(1 / m)\left[B_{0}+\right.$ $\left.\sqrt{B_{0}^{2}-4 h m}\right], Q_{1}^{2}=(1 / m)\left[-B_{0}+\sqrt{B_{0}^{2}-4 h m}\right]$, and $h \in$ $\left(-B_{0}^{2} / 4 m,+\infty\right)$. Without loss of generality, we suppose that $P_{1}>Q_{1}>0$. Define $\varphi / P_{1}=t$ and $k^{2}=\left(P_{1}^{2}-Q_{1}^{2}\right) / P_{1}^{2}$; hence, (9) becomes

$$
\left(t^{\prime}\right)^{2}=-\frac{m P_{1}^{2}}{2}\left(1-t^{2}\right)\left(t^{2}-1+k^{2}\right) .
$$

Next, we continue to define a new variable $r$, which satisfies $r \geq 0$ and $r(0)=0$. By relation $t^{2}=1-k^{2} \sin ^{2} r$, we obtain

$$
\left(r^{\prime}\right)^{2}=\frac{-m P_{1}^{2}}{2}\left(1-k^{2} \sin ^{2} r\right) .
$$

So, we get for $s=\sqrt{-m / 2} P_{1}$ that

$$
\int_{0}^{\gamma(\xi)} \frac{d t}{\sqrt{1-k^{2} \sin ^{2} 2}}=s \xi
$$

Next, inspired by Angulo Pava's work [12], by applying the theory of Jacobian elliptic function [13], we obtain the dnoidal wave solution of (8),

$$
\varphi(\xi)=P_{1} d n\left(\sqrt{-\frac{m}{2}} P_{1} \xi, \frac{\sqrt{P_{1}^{2}-Q_{1}^{2}}}{P_{1}}\right),
$$

where $\alpha=\sum_{j=1}^{n} \alpha_{j}^{2}$ and $\beta=\sum_{j=1}^{n} \beta_{j}^{2}$. 
with

$$
\begin{aligned}
k^{2} & =\frac{P_{1}^{2}-Q_{1}^{2}}{P_{1}^{2}}, \\
P_{1}^{2}+Q_{1}^{2} & =-\frac{2 B_{0}}{m} .
\end{aligned}
$$

Because $\mathrm{dn}$ has fundamental period $2 K$; that is, $d n(x+$ $2 K ; k)=d n(x, k)$. Here $K(k)$ is the complete elliptic integral of first kind. Hence, we know that solution (13) with fundamental period is as follows:

$$
T=\frac{2 K(k)}{\sqrt{-m / 2} P_{1}} .
$$

Next, expression (15) can be converted into a simple form according to (14). In fact, if we fix variables $m$ and $B_{0}$, we can find (15) as a function with only one variable $Q_{1}$ :

$$
\begin{aligned}
T\left(Q_{1}\right) & =\frac{2 K\left(k\left(Q_{1}\right)\right)}{\sqrt{B_{0}+(m / 2) Q_{1}^{2}}}, \\
k^{2}\left(Q_{1}\right) & =\frac{-2 B_{0} / m-2 Q_{1}^{2}}{-2 B_{0} / m-Q_{1}^{2}} .
\end{aligned}
$$

Furthermore, if $Q_{1} \rightarrow 0, k\left(Q_{1}\right) \rightarrow 1$, and $K\left(k\left(Q_{1}\right)\right) \rightarrow$ $+\infty, T\left(Q_{1}\right) \rightarrow+\infty$. If $Q_{1} \rightarrow \sqrt{-B_{0} / m}, k\left(Q_{1}\right) \rightarrow 0$, and $k\left(Q_{1}\right) \rightarrow \pi / 2$, therefore, $T\left(Q_{1}\right) \rightarrow \pi / \sqrt{B_{0}}$.

Then, we will establish a smooth $\mathrm{dn}$ wave solution for (8) with fixed period $L$. For any given $L>\pi / \sqrt{B_{0}}, B_{0}>$ $\pi^{2} / L^{2}$, and $m<0$, we can know that the mapping $Q_{1} \in$ $\left(0, \sqrt{-B_{0} / m}\right) \rightarrow T\left(Q_{1}\right)$ is strictly decreasing function. In what follows, we will show that there is a unique $Q_{1}=$ $Q_{1}\left(B_{0}\right) \in\left(0, \sqrt{-B_{0} / m}\right)$ such that $T\left(Q_{1}\right)=T\left(Q_{1}\left(B_{0}\right)\right)$ is a fundamental period of (13).

Theorem 1. For any given $L>\pi / \sqrt{B_{0}}$, consider $B_{0,0}>\pi^{2} / L^{2}$; then there is a unique $Q_{1,0} \in\left(0, \sqrt{-B_{0,0} / m}\right)$ such that $T_{\left(B_{0,0}\right)}=$ L. Then,

(1) there exist an interval $I\left(B_{0,0}\right)$ around $B_{0,0}$, an interval $C\left(Q_{1,0}\right)$ around $Q_{1,0}$, and a smooth and unique function $\Upsilon: I\left(B_{0,0}\right) \rightarrow C\left(Q_{1,0}\right)$, such that $\Upsilon\left(B_{0,0}\right)=Q_{1,0}$ and

$$
T\left(Q_{1}\right)=\frac{2 K(k)}{\sqrt{B_{0}+(m / 2) Q_{1}^{2}}} .
$$

Here, $B_{0} \in I\left(B_{0,0}\right)$ and $Q_{1}=\Upsilon\left(B_{0}\right)$.

(2) The solution $\varphi(\xi)=P_{1} d n\left(\sqrt{-m / 2} P_{1} \xi, \sqrt{P_{1}^{2}-Q_{1}^{2}} / P_{1}\right)$ is determined by $P_{1}=P_{1}\left(B_{0}\right)$ and $Q_{1}=Q_{1}\left(B_{0}\right)$, has fundamental period $L$, and satisfies (13). In addition, the mapping $B_{0} \in I\left(B_{0,0}\right) \rightarrow \varphi_{B_{0}} \in H_{\text {per }}^{1}([0, L])$ is a smooth function.

Proof. The proof is via the implicit function theorem, the method is based on Angulo Pava's work [12], and for more details, one may see Theorem 1 in [12].
Theorem 2. Let $L>0$. Then, there is a smooth curve of periodic traveling wave solutions for (8) with $B_{0} \in$ $(0,+\infty) \rightarrow \varphi_{B_{0}} \in H_{\text {per }}^{1}([0, L])$. Here, $n \in N, \varphi(\xi)=$ $P_{1} d n\left(\sqrt{-m / 2} P_{1} \xi, \sqrt{P_{1}^{2}-Q_{1}^{2}} / P_{1}\right)$.

\section{Spectral Analysis}

Firstly, (1) can be rewritten as the following form:

$$
\begin{aligned}
& \phi_{t}=\widetilde{\phi}, \\
& \widetilde{\phi}_{t}=\Delta \phi-m_{1} \phi+\left(a_{11}|\phi|^{2}+a_{12}|\psi|^{2}\right) \phi, \\
& \psi_{t}=\widetilde{\psi}, \\
& \widetilde{\psi_{t}}=\Delta \psi-m_{2} \psi+\left(a_{21}|\phi|^{2}+a_{22}|\psi|^{2}\right) \psi .
\end{aligned}
$$

Moreover, let $\vec{U}=(\phi, \widetilde{\phi}, \psi, \widetilde{\psi})^{T}$. So, (1) can be rewritten as the following Hamiltonian system:

$$
\frac{d \vec{U}}{d t}=J E^{\prime}(\vec{U}),
$$

where $J$ is the skew-symmetric linear operator:

$$
\begin{aligned}
J & =\left(\begin{array}{cccc}
0 & -1 & 0 & 0 \\
1 & 0 & 0 & 0 \\
0 & 0 & 0 & -1 \\
0 & 0 & 1 & 0
\end{array}\right), \\
E(\vec{U})= & \frac{1}{2} \int_{0}^{L}\left(|\widetilde{\phi}|^{2}-|\nabla \cdot \phi|^{2}-m_{1}|\phi|^{2}+\frac{1}{2} a_{11}|\phi|^{4}\right. \\
& +a_{12}|\psi|^{2}|\phi|^{2}+|\widetilde{\psi}|^{2}-|\nabla \cdot \psi|^{2}-m_{2}|\psi|^{2} \\
& \left.+\frac{1}{2} a_{22}|\psi|^{4}\right) d x .
\end{aligned}
$$

Let $T$ be a one-parameter group of unitary operator on $X$ defined by

$$
T(s) \vec{U}(\cdot)=\left(\phi(\cdot) e^{i s}, \widetilde{\phi}(\cdot) e^{i s}, \psi(\cdot) e^{i s}, \widetilde{\psi} e^{i s}\right),
$$

where $\vec{U}(\cdot) \in X$ and $s \in R$.

Obviously,

$$
T^{\prime}(0)=\left(\begin{array}{cccc}
i & 0 & 0 & 0 \\
0 & i & 0 & 0 \\
0 & 0 & i & 0 \\
0 & 0 & 0 & i
\end{array}\right) .
$$

Since $J B=T^{\prime}(0)$, we can deduce that

$$
B=\left(\begin{array}{cccc}
0 & i & 0 & 0 \\
-i & 0 & 0 & 0 \\
0 & 0 & 0 & -i \\
0 & 0 & i & 0
\end{array}\right) \text {. }
$$


Next, we define the functional

$$
\begin{aligned}
& Q(\vec{U})=\frac{1}{2} \int_{0}^{L}(|\phi \widetilde{\phi}|+|\widetilde{\psi} \psi|) d x, \\
& H(\vec{U})=E(\vec{U})-c Q(\vec{U}) .
\end{aligned}
$$

According to [5], it can be verified that $E(\vec{U})$ and $Q(\vec{U})$ are conserved quantities, and the solutions of system (1) are critical points of $H(\vec{U})$; namely,

$$
H^{\prime}(\vec{U})=0 .
$$

Next, we define an operator $L$ from $X$ to $X^{*}$ :

$$
L=H^{\prime \prime}(\vec{U})=E^{\prime \prime}(\vec{U})-c Q^{\prime \prime}(\vec{U})
$$

$$
L \vec{\zeta}=\left(\begin{array}{l}
\Delta \zeta_{1}-m_{1} \zeta_{1}+a_{11}|\phi|^{2} \zeta_{1}+2 a_{12} \phi^{2} \zeta_{1}+a_{12}|\psi|^{2} \zeta_{1}-c \zeta_{2}+2 a_{12} \psi \phi \zeta_{3}-c \zeta_{1}+\zeta_{2} \\
\Delta \zeta_{3}-m_{2} \zeta_{3}+2 a_{12} \phi \psi \zeta_{1}+a_{12}|\phi|^{2} \zeta_{3}+a_{22}|\psi|^{2} \zeta_{3}+2 a_{22} \psi^{2} \zeta_{3}-c \zeta_{4}-c \zeta_{3}+\zeta_{4}
\end{array}\right) .
$$

By a simple computation, we can deduce that $L$ is a selfadjoint operator. It is shown that $I^{-1} L$ is a bounded selfadjoint operator. The spectrum of the $L$ consists of the real numbers $\lambda$ such that $\lambda I-L$ is not invertible, and $\lambda=0$ belongs to the spectrum of $L$.

Moreover, we can also prove that

$$
L T^{\prime}(0) \vec{U}=0 .
$$

Let

$$
Z=\left\{k \in R \mid k T^{\prime}(0) \vec{U}\right\} .
$$

From (28), we can know that $Z$ is contained in the kernel of $L$.

By [14], we have the following theorem.

Theorem 3. The space $X$ is decomposed as a direct sum; $X=$ $Z+N+P$. Here, $Z$ is defined by (29), $N$ is a finite-dimensional subspace, such that

$$
\langle L \vec{u}, \vec{u}\rangle<0, \quad \text { for } 0 \neq \vec{u} \in N,
$$

and $P$ is a closed subspace, such that

$$
\langle L \vec{u}, \vec{u}\rangle \geq \sigma\|\vec{u}\|_{X}^{2}, \quad \text { for } \vec{u} \in P,
$$

where $\sigma$ is a positive number and independent of $\vec{u}$.

Proof. For any $\omega \in X$, let

$$
\omega=\left(\omega_{1}, \omega_{2}, \omega_{3}, \omega_{4}\right)^{T} .
$$

Then,

$$
\begin{aligned}
\langle L \omega, & \omega\rangle \\
= & \left\langle L_{1} \omega_{1}, \omega_{1}\right\rangle+\left\langle L_{2} \omega_{3}, \omega_{3}\right\rangle \\
& +2 \operatorname{Re} \int_{R}\left(-c \overline{\omega_{1}} \omega_{2}+2 a_{12} \psi \phi \omega_{3} \overline{\omega_{1}}-c \overline{\omega_{3}} \omega_{4}\right) d x \\
& +\left\langle\omega_{2}+\omega_{4}, \omega_{2}+\omega_{4}\right\rangle-\left\langle c^{2} \omega_{1}-\alpha \omega_{1}, \omega_{1}\right\rangle \\
& -\left\langle c^{2} \omega_{3}-\beta \omega_{3}, \omega_{3}\right\rangle,
\end{aligned}
$$

where

$$
\begin{aligned}
& L_{1}=\Delta-m_{1}+a_{11}|\phi|^{2}+a_{12}|\psi|^{2}+c^{2}-\alpha, \\
& L_{2}=\Delta-m_{2}+a_{12}|\phi|^{2}+2 a_{22} \psi^{2}+c^{2}-\beta .
\end{aligned}
$$

As $|x| \rightarrow \infty, \phi, \psi \rightarrow 0$. So that

$$
\begin{aligned}
& \sigma_{\mathrm{ess}}\left(L_{1}\right)=\left[-m_{1}+c^{2}-\alpha,+\infty\right), \\
& \sigma_{\mathrm{ess}}\left(L_{2}\right)=\left[-m_{2}+c^{2}-\beta,+\infty\right) .
\end{aligned}
$$

Moreover, we may obtain that

$$
\begin{aligned}
& L_{1} u=0, \\
& L_{2} \varphi=0 .
\end{aligned}
$$

From $(13)$ and $[5,15]$, they imply $u$ has a simple zero. By Sturm-Liouville theorem, 0 is the second eigenvalue of $L_{1}$, and $L_{1}$ has exactly one strictly negative eigenvalue $-\sigma_{1}^{2}$, with an eigenfunction $\chi$; that is,

$$
L_{1} \chi=-\sigma_{1}^{2} \chi .
$$

Furthermore, 0 is the first simple eigenvalue of $L_{2}$. From [16], we have the following lemmas (Lemmas 4 and 5).

Lemma 4. For any real function $z_{1} \in H(R)$, if it satisfies

$$
\left\langle z_{1}, \chi\right\rangle=\left\langle z_{1}, u\right\rangle=0 .
$$

There exists a number $\sigma_{1} \in R^{+}$independent of $z_{1}$, such that

$$
\left\langle L_{1} z_{1}, z_{1}\right\rangle \geq \sigma_{1}\left\|z_{1}\right\|_{H^{1}}^{2} .
$$

Lemma 5. For any real function $z_{2} \in H(R)$, if it satisfies

$$
\left\langle z_{2}, \varphi\right\rangle=0 .
$$


Then, there exits a number $\sigma_{2} \in R^{+}$independent of $z_{2}$, such that

$$
\left\langle L_{2} z_{2}, z_{2}\right\rangle \geq \sigma_{2}\left\|z_{2}\right\|_{H^{1}}^{2} .
$$

Let $\vec{y}=\left(\chi_{1}, 0, \varphi, 0\right)^{T} \in X$. By (3), we can obtain

$$
\langle L \vec{y}, \vec{y}\rangle=-\sigma_{1}^{2}\langle\vec{y}, \vec{y}\rangle .
$$

Notice that the kernel of $L$ is spanned by $\overrightarrow{v_{1}}$ and $\overrightarrow{v_{2}}$; then

$$
Z=\left\{k_{1} \overrightarrow{v_{1}}+k_{2} \overrightarrow{v_{2}} \mid k_{1}, k_{2} \in R\right\} \text {. }
$$

Let

$$
\begin{aligned}
\vec{P} & =\left\{\vec{p} \in X \mid\langle\vec{p}, \vec{y}\rangle=\left\langle\vec{p}, \overrightarrow{v_{1}}\right\rangle=\left\langle\vec{p}, \overrightarrow{v_{2}}\right\rangle=0\right\}, \\
N & =\left\{k_{2} \vec{y} \mid k_{2} \in R\right\} .
\end{aligned}
$$

For any $\omega \in X$ and $\vec{p} \in P$, we choose $a_{1}=\langle\vec{p}, \vec{y}\rangle /\langle\vec{y}, \vec{y}\rangle$, $a_{2}=\left\langle\vec{p}, \overrightarrow{v_{1}}\right\rangle /\left\langle\overrightarrow{v_{1}}, \overrightarrow{v_{1}}\right\rangle$, and $a_{3}=\left\langle\vec{p}, \overrightarrow{v_{2}}\right\rangle /\left\langle\overrightarrow{v_{2}}, \overrightarrow{v_{2}}\right\rangle$. Then $\omega$ can be uniquely represented by

$$
\omega=a_{1} \vec{y}+a_{2} \overrightarrow{v_{1}}+a_{3} \overrightarrow{v_{2}}+\vec{p} .
$$

This shows that the space $X$ is decomposed as a direct sum; $X=Z+N+P$.

In the following, we will prove that

$$
\forall \vec{p} \in P, \quad\langle L \vec{p}, \vec{p}\rangle \geq \sigma\|\vec{p}\|_{H^{1}}^{2} .
$$

Let $\vec{p}=\left(p_{1}, p_{2}, p_{3}, p_{4}\right)^{T}$. By Lemmas 4 and 5 , we have

$$
\begin{aligned}
\langle L \vec{p}, \vec{p}\rangle \geq & \delta_{1}\left\|p_{1}\right\|_{H^{1}}^{2}+\delta_{2}\left\|p_{3}\right\|_{H^{1}}^{2} \\
& -c\left(\left\|\overline{p_{1}}\right\|_{H^{1}}^{2}+\left\|\overline{p_{2}}\right\|_{H^{1}}^{2}\right) \\
& -c\left(\left\|\overline{p_{3}}\right\|_{H^{1}}^{2}+\left\|\overline{p_{4}}\right\|_{H^{1}}^{2}\right)+\left\|\overline{p_{2}}\right\|_{H^{1}}^{2}+\left\|\overline{p_{4}}\right\|_{H^{1}}^{2} \\
& -\left(c^{2}-\alpha\right)\left\|p_{1}\right\|_{H^{1}}^{2}-\left(c^{2}-\beta\right)\left\|p_{3}\right\|_{H^{1}}^{2} \\
\geq & \delta\left(\left\|p_{1}\right\|_{H^{1}}^{2}+\left\|p_{2}\right\|_{H^{1}}^{2}+\left\|p_{3}\right\|_{H^{1}}^{2}+\left\|p_{4}\right\|_{H^{1}}^{2}\right),
\end{aligned}
$$

where $\delta=\min \left\{\alpha+\delta_{1}-2 c^{2}, 1-c, \beta+\delta_{2}-2 c^{2}\right\}$. Thus, we complete the proof of Theorem 3 .

\section{Orbital Stability of the Periodic Wave Solutions for the $(n+1)$-Dimensional KG Equations}

In this section, we shall apply the method established by Grillakis et al. [5] to give the orbital stability of the periodic traveling solutions of system (1):

$$
\vec{\Phi}(x, t)=(\phi, \psi)=\left(e^{i \eta_{1}} a \varphi(\xi), e^{i \eta_{2}} \varphi(\xi)\right),
$$

where

$$
\varphi(\xi)=P_{1} d n\left(\sqrt{\frac{-m}{2}} P_{1} \xi, \frac{\sqrt{P_{1}^{2}-Q_{1}^{2}}}{P_{1}}\right)
$$

By $[16,17]$ and by applying classical semigroups method, for any

$$
\begin{aligned}
\vec{\Phi}(0) & =(\phi(x, 0), \psi(x, 0)) \\
& \in H_{\text {per }}^{1}\left(R^{n},[0, L]\right) \times H_{\text {per }}^{1}\left(R^{n},[0, L]\right),
\end{aligned}
$$

there exists a solution $\vec{\Phi}(x, t) \in H_{\text {per }}^{1}\left(R^{n},[0, L]\right) \times$ $H_{\text {per }}^{1}\left(R^{n},[0, L]\right)$ for system (1) and $L=+\infty$ or $L<+\infty$.

In Section 2, we have obtained that system (1) has periodic traveling waves solution of $(48)$ in $H_{\text {per }}^{1}[0, L] \times H_{\text {per }}^{1}[0, L]$. Moreover, in Section 3, we also obtained that operator $L$ has only one negative simple eigenvalue, the kernel of $L$ is spanned by $T^{\prime}(0) \Phi$, and the rest of its spectrum is positive and bounded away from zero. According to orbital stability theory of Grillakis et al. [6], we only need to prove that the function is strictly convex as follows:

$$
d(c)=E(\vec{U})-c \mathrm{Q}(\vec{U}) .
$$

Firstly, we state our definition of orbital stability.

Definition 6. The orbit of $\vec{\Phi}(x, t)=(\phi, \psi)$ is defined by

$$
\Omega_{\vec{\Phi}}:=\left\{T(s) \vec{\Phi}:=\vec{\Phi} e^{i s}, s \in R^{2}\right\} .
$$

We say orbit $\Omega_{\vec{\Phi}}$ is orbital stable in Banach space $X$, if for all $\varepsilon>0$ there exists a $\delta>0$ such that if $\vec{\Phi}(x, 0)=$ $(\phi(x, 0), \psi(x, 0)) \in X=H_{\text {per }}^{1}\left(R^{n},[0, L]\right) \times H_{\text {per }}^{1}\left(R^{n},[0, L]\right)$ satisfies $\|\vec{\Phi}(0)-\vec{\Phi}\|_{X}<\delta$, and $\vec{\Phi}(x, t)$ is a solution of (1) in internal $\left[0, t_{0}\right)$ with the initial value $\vec{\Phi}(x, 0)=\vec{\Phi}(0)$, then $\vec{\Phi}(x, t)$ can be extended to a solution in $0 \leq t<+\infty$ has

$$
\sup _{t>0} \inf _{s \in R}\|\vec{\Phi}(t)-T(s) \vec{\Phi}\|_{X}<\varepsilon .
$$

Otherwise, we say that $\vec{\Phi}(x, t)$ is orbital unstable.

Theorem 7. Let $a c+1<0$ and $\vec{\Phi}(x, t)=\left(a e^{\eta_{1}} \varphi(\xi), e^{\eta_{2}} \varphi(\xi)\right)$, $\varphi(\xi)$ determined by (49), be a periodic traveling waves solution of system (1). Then, this solution is orbital stable in $H_{\text {per }}^{1}\left(R^{n},[0, L]\right) \times H_{p e r}^{1}\left(R^{n},[0, L]\right)$ by periodic flow of system (1).

Proof. From (3), we know that

$$
E^{\prime}(\vec{U})-c Q^{\prime}(\vec{U})=0
$$

Therefore

$$
\begin{aligned}
d^{\prime}(c) & =E^{\prime}(\vec{U})-c Q^{\prime}(\vec{U})-Q(\vec{U})=-Q(\vec{U}) \\
& =-(a c+1) \int_{0}^{L}(\varphi(\xi))^{2} d \xi
\end{aligned}
$$

Then

$$
d^{\prime \prime}(c)=-\frac{d}{d c}\left((a c+1) \int_{0}^{L}(\varphi(\xi))^{2} d \xi\right)
$$


Through (13), we obtain that

$$
\begin{aligned}
\|\varphi\|^{2} & =P_{1}^{2} \int_{0}^{L} d n^{2}\left(\sqrt{-\frac{m}{2}} P_{1} \xi, k\right) d \xi \\
& =P_{1}^{2} \int_{0}^{\sqrt{-m / 2} P_{1} L} d n^{2}(\xi, k) d \xi \\
& =\frac{P_{1}}{\sqrt{-m / 2}} \int_{0}^{2 K} d n^{2}(\xi, k) d \xi \\
& =2 \frac{K}{\sqrt{-m / 2} L} \int_{0}^{K} d n^{2}(\xi, k) d \xi .
\end{aligned}
$$

Now, by using that (see [13]),

$$
\begin{aligned}
\int_{0}^{K} c n^{2}(\xi ; k) d \xi & =\frac{1}{k^{2}}\left[E(k)-\left(1-k^{2}\right) K(k)\right], \\
d n^{2}(\xi ; k) & =1-k^{2}+k^{2} c n^{2}(\xi ; k) .
\end{aligned}
$$

Therefore, we can obtain that

$$
\int_{0}^{L} \varphi^{2}(\xi) d \xi=\frac{4 K E}{L} \frac{1}{-m / 2} .
$$

So that

$$
\begin{aligned}
d^{\prime \prime}(c) & \\
= & -\left(a \int_{0}^{L} \varphi^{2}(\xi) d \xi+(a c+1) \frac{d}{d c}\left(\int_{0}^{L} \varphi^{2}(\xi) d \xi\right)\right) \\
= & -\left(a \frac{4 K E}{L} \frac{1}{-m / 2}\right) \\
& +(a c+1)\left(-\frac{1}{L} \frac{1}{2 m} \frac{d}{d k}(4 E K) \frac{d B_{0}}{d c} \frac{d k}{d B_{0}}\right) \\
= & -\left(a \frac{4 K E}{L} \frac{1}{-m / 2}\right) \\
& +(a c+1)\left(-\frac{1}{L} \frac{1}{2 m} \frac{d}{d k}(4 E K)(-2 c) \frac{d k}{d B_{0}}\right) .
\end{aligned}
$$

Indeed,

$$
k^{2}=\frac{P_{1}^{2}-Q_{1}^{2}}{P_{1}^{2}}=\frac{2 \sqrt{B_{0}^{2}-4 h m}}{B_{0}^{2}+\sqrt{B_{0}^{2}-4 h m}} .
$$

So that

$$
\frac{d k}{d B_{0}}=\frac{4 h m}{\left(\sqrt{B_{0}^{2}-4 h m}+B_{0}\right)^{2} \sqrt{B_{0}^{2}-4 h m}}<0
$$

Moreover, according to [13], $E(k) K(k)$ is strictly increasing functions; hence, $d(E(k) K(k)) / d k>0$. Hence, we can obtain $d^{\prime \prime}(c)>0$. So, we complete the proof of this theorem.

\section{Discussion and Conclusion}

In this paper, inspired by Angulo Pava's ideas [11, 12, 18, 19], and by using the stability theory established by Grillakis et al. [5], we obtain solution (48) of system (1) and prove that the solution is orbital stable. The method is helpful to look for periodic solution and obtain the orbital stability for a class of nonlinear equations, which is widely used in quantum mechanics, fluid mechanics, and optical fiber communication. Hence, it may contribute to solving these engineering problems.

\section{Conflict of Interests}

The authors declare that there is no conflict of interests regarding the publication of this paper.

\section{References}

[1] E. Fan, "Extended tanh-function method and its applications to nonlinear equations," Physics Letters A, vol. 277, no. 4-5, pp. 212-218, 2000.

[2] E. J. Parkes and B. R. Duffy, "Travelling solitary wave solutions to a compound KdV-Burgers equation," Physics Letters A, vol. 229, no. 4, pp. 217-220, 1997.

[3] Z. Fu, S. Liu, S. Liu, and Q. Zhao, "New Jacobi elliptic function expansion and new periodic solutions of nonlinear wave equations," Physics Letters A, vol. 290, no. 1-2, pp. 72-76, 2001.

[4] S. Liu, Z. Fu, S. Liu, and Q. Zhao, "Jacobi elliptic function expansion method and periodic wave solutions of nonlinear wave equations," Physics Letters A, vol. 289, no. 1-2, pp. 69-74, 2001.

[5] M. Grillakis, J. Shatah, and W. Strauss, "Stability theory of solitary waves in the presence of symmetry. I," Journal of Functional Analysis, vol. 74, no. 1, pp. 160-197, 1987.

[6] M. Grillakis, J. Shatah, and W. Strauss, "Stability theory of solitary waves in the presence of symmetry, II," Journal of Functional Analysis, vol. 94, no. 2, pp. 308-348, 1990.

[7] T. B. Benjamin, “The stability of solitary waves," Proceedings of the Royal Society of London Series A: Mathematical and Physical Sciences, vol. 328, no. 1573, pp. 153-183, 1972.

[8] M. I. Weinstein, "Lyapunov stability of ground states of nonlinear dispersive evolution equations," Communications on Pure and Applied Mathematics, vol. 39, no. 1, pp. 51-67, 1986.

[9] J. Shatah, "Stable standing waves of nonlinear Klein-Gordon equations," Communications in Mathematical Physics, vol. 91, no. 3, pp. 313-327, 1983.

[10] M. Ohta and G. Todorova, "Strong instability of standing waves for nonlinear Klein-Gordon equations," Discrete and Continuous Dynamical Systems. Series A, vol. 12, no. 2, pp. 315322, 2005.

[11] J. Angulo Pava, "Stability of cnoidal waves to Hirota-Satsuma systems," Matemática Contemporânea, vol. 27, pp. 189-223, 2004.

[12] J. Angulo Pava, "Nonlinear stability of periodic traveling wave solutions to the Schrödinger and the modified Korteweg-de Vries equations," Journal of Differential Equations, vol. 235, no. 1, pp. 1-30, 2007. 
[13] P. F. Byrd and M. D. Friedman, Handbook of Elliptic Integrals for Engineers and Scientists, Springer, New York, NY, USA, 2nd edition, 1971.

[14] W. Zhang, Y. Qin, Y. Zhao, and B. Guo, "Orbital stability of solitary waves for Kundu equation," Journal of Differential Equations, vol. 247, no. 5, pp. 1591-1615, 2009.

[15] J. P. Albert and J. L. Bona, "Total positivity and the stability of internal waves in stratified fluids of finite depth," IMA Journal of Applied Mathematics, vol. 46, no. 1-2, pp. 1-19, 1991.

[16] I. Segal, "Non-linear semi-groups," Annals of Mathematics. Second Series, vol. 78, pp. 339-364, 1963.

[17] V. Georgiev, "Global solution of the system of wave and KleinGordon equations," Mathematische Zeitschrift, vol. 203, no. 4, pp. 683-698, 1990.

[18] J. Angulo, Nonlinear Dispersive Equations. Existence and Stability of Solitary and Periodic Travelling Wave Solutions, vol. 156 of Mathematical Surveys and Monographs, American Mathematical Society, Providence, RI, USA, 2009.

[19] J. A. Pava and F. Natali, "(Non)linear instability of periodic traveling waves: klein-Gordon and KDV type equations," Advances in Nonlinear Analysis, vol. 3, no. 2, pp. 95-123, 2014. 


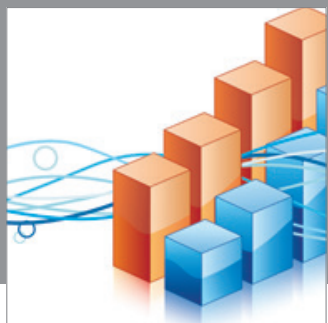

Advances in

Operations Research

mansans

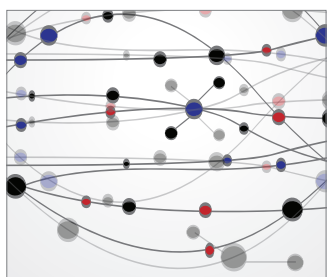

The Scientific World Journal
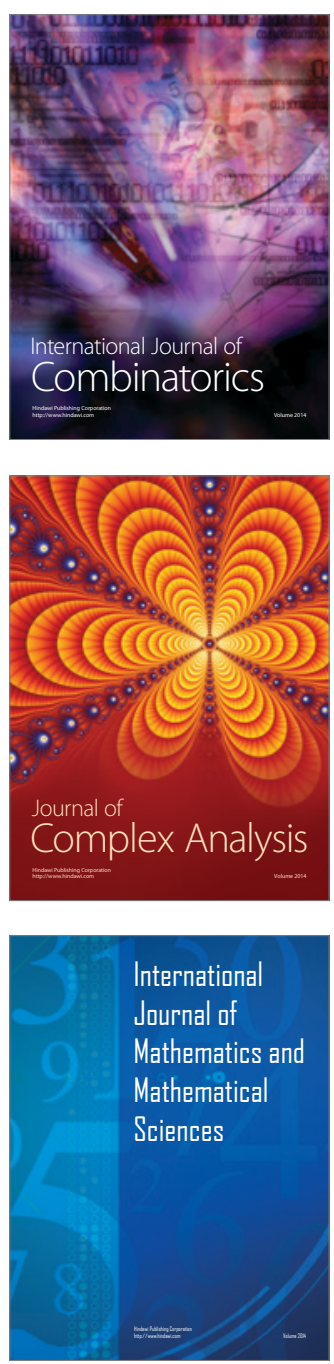
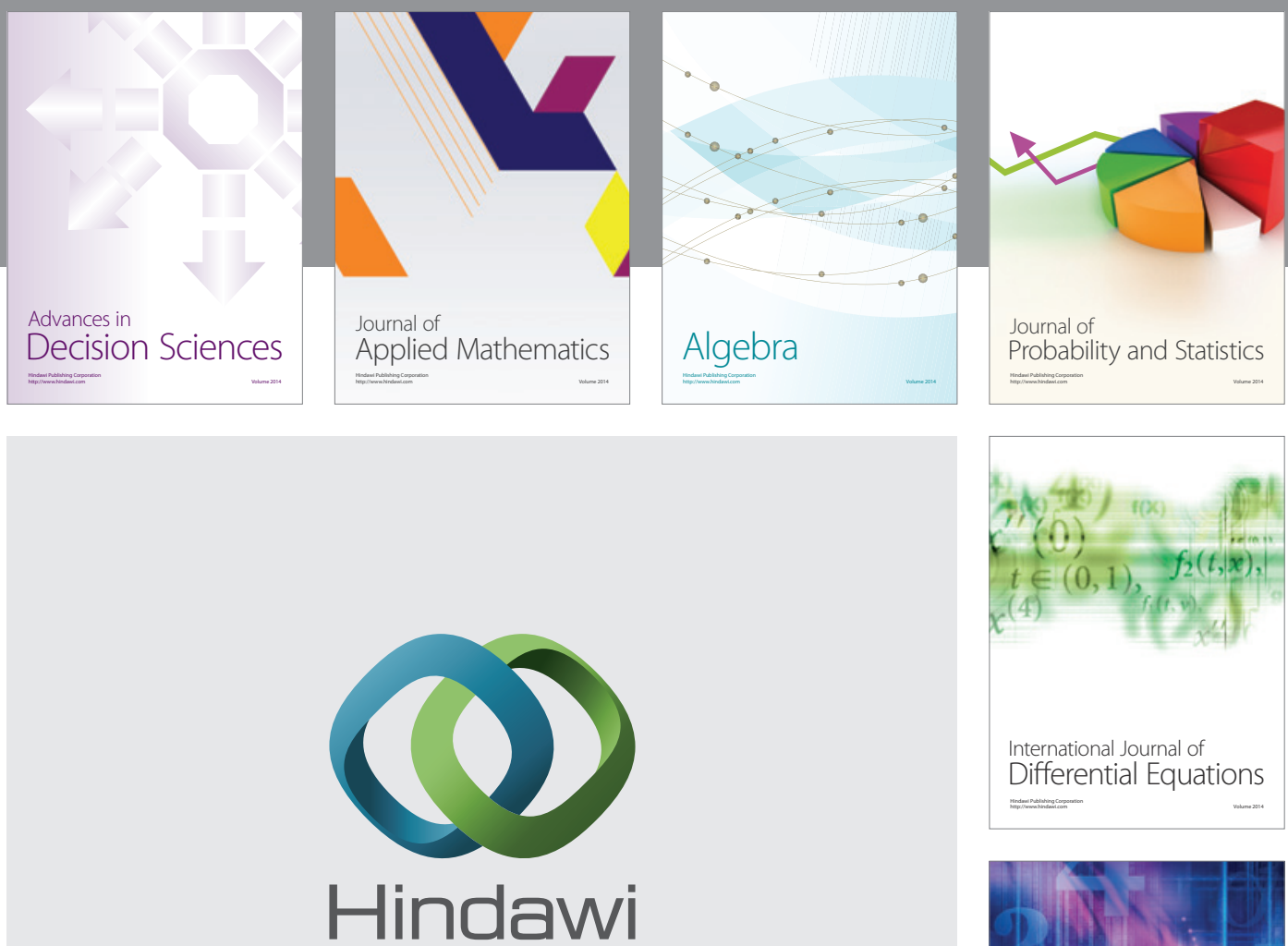

Submit your manuscripts at http://www.hindawi.com
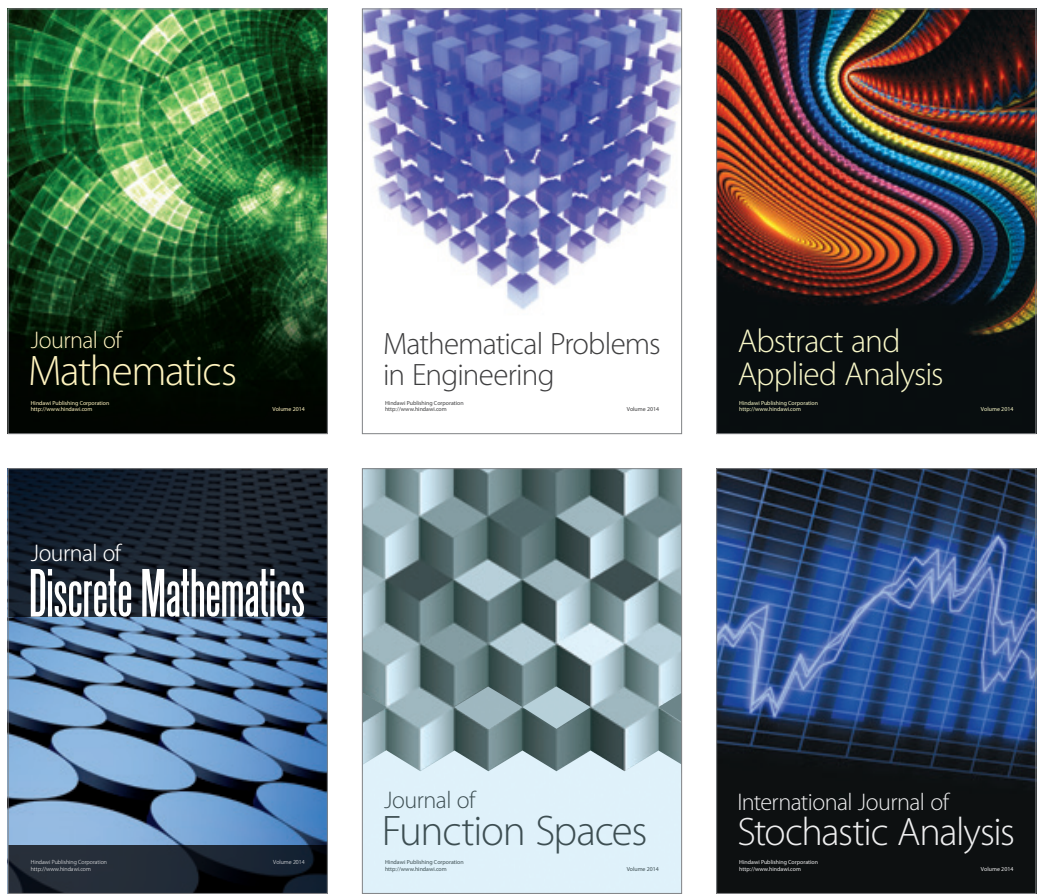

Journal of

Function Spaces

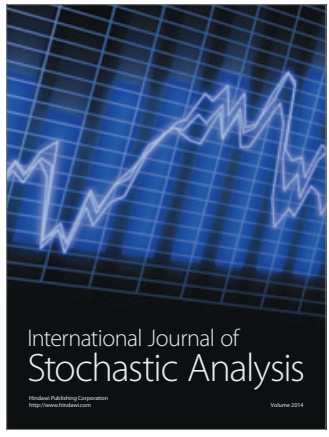

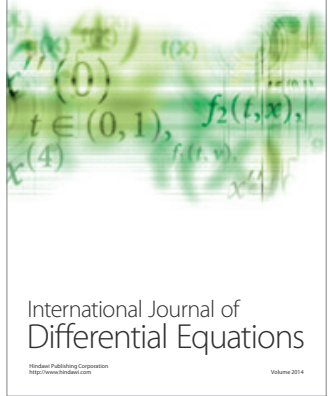
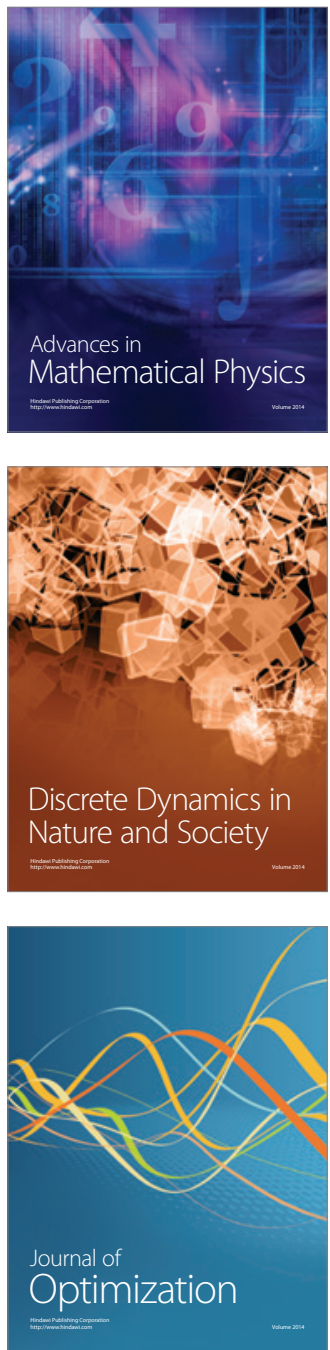